Математички Билтен

ISSN 0351-336X (print)

37(LXIII) No. 1

ISSN 1857-9914 (online)

2013(61-74)

UDC: 519.173 .174

Скопје, Македонија

\title{
WEAK-ODD EDGE-COLORING OF DIGRAPHS
}

\author{
MIRKO PETRUŠEVSKI AND RISTE ŠKREKOVSKI
}

\begin{abstract}
A weak-odd edge-coloring of a digraph $D$ is a (not necessarily proper) edge-coloring such that for each vertex $v \in V(D)$ at least one color $c$ satisfies the following requirement: if $d^{+}(v)>0$ then $c$ appears an odd number of times on the outgoing edges at $v$; and if $d^{-}(v)>0$ then $c$ appears an odd number of times on the ingoing edges at $v$. The minimum number of colors sufficient for a weak-odd edge-coloring of $D$ is the weak-odd chromatic index, denoted $\chi_{\text {wo }}^{\prime}(D)$. In this article we prove that $\chi_{\text {wo }}^{\prime}(D) \leq 3$ for every digraph $D$, and show that this bound is sharp. We study when does a graph admit an orientation so that the obtained digraph is weak-odd 1-edge-colorable. We also prove that every graph admits an orientation for which the obtained digraph is weak-odd 2-edge-colorable.
\end{abstract}

\section{INTRODUCTION}

1.1. Terminology and notation. Throughout the article we mainly follow terminology and notation used in $[1,7]$. All considered digraphs and graphs are finite (i.e. have finite sets of vertices and edges). A directed graph (or digraph) $D$ is a triple consisting of a vertex set $V(D)$, an edge set $E(D)$, and a map which assigns to each edge an ordered pair of vertices: the first vertex of the ordered pair is the tail of the edge, and the second is the head; together they are the endpoints. Thus each edge is said to be directed from its tail to its head. An edge with tail $u$ and head $v$ is referred to as a directed uv-edge (the adjective 'directed' is often omitted). Given a vertex $v \in V(D)$, an outgoing (resp. ingoing) edge at $v$ is any edge having tail (resp. head) $v$. Denote by $E_{D}^{+}(v)$ (resp. $E_{D}^{-}(v)$ ) the set of outgoing (resp. ingoing) edges at $v$. A loop is an edge of a digraph whose endpoints are equal. Each loop at a vertex $v$ belongs to both $E_{D}^{+}(v)$ and $E_{D}^{-}(v)$. Parallel edges are edges having the same ordered pair of endpoints. The size of the set $E_{D}^{+}(v)$ (resp. $E_{D}^{-}(v)$ ) is called the outdegree $d_{D}^{+}(v)$ (resp. indegree $\left.d_{D}^{-}(v)\right)$ of the vertex $v$. If $d_{D}^{+}(v)=0\left(\right.$ resp. $\left.d_{D}^{-}(v)=0\right)$, then $v$ is called a

2000 Mathematics Subject Classification. 05C10.

Key words and phrases. digraph, weak-odd edge-coloring, weak-odd chromatic index. 
sink (resp. source) of $D$. Any sink or source is a peripheral vertex of $D$. On the other hand, any vertex that is neither a source nor a sink is an intermediate vertex of $D$. For $v \in V(D)$, the union $E_{D}(v)=E_{D}^{+}(v) \cup E_{D}^{-}(v)$ is the set of edges incident to $v$, and the sum $d_{D}(v)=d_{D}^{+}(v)+d_{D}^{-}(v)$ is the degree of $v$. Whenever $d_{D}(v)=1$ we say that $v$ is a pendant vertex, and then its only incident edge is a pendant edge of $D$. For $X \subseteq V(D) \cup E(D)$, denote by $D-X$ the subdigraph of $D$ obtained by removing $X$. The underlying graph of a digraph $D$ is the graph $G$ acquired by 'forgeting' the direction of each edge, i.e. by treating the edges as unordered pairs: the vertex set and the edge set remain the same, the endpoints of every edge are the same in $G$ as in $D$, but in $G$ they become an unordered pair. We say that a digraph $D$ is connected if its underlying graph is connected.

Similar to digraphs, for a given graph $G$ and a vertex $v \in V(G)$, the subset of $E(G)$ consisting of the edges incident to $v$ is denoted by $E_{G}(v)$. For every $v \in V(G)$, the size of the set $E_{G}(v)$ is the degree $d_{G}(v)$ of $v$ (recall that every loop at $v$ is counted twice for $\left.\left|E_{G}(v)\right|\right)$. We refer to each vertex $v \in V(G)$ of even (resp. odd) degree $d_{G}(v)$ as an even (resp. odd) vertex of $G$. A graph is called even (resp. odd) whenever all its vertices are even (resp. odd).

1.2. Definition of weak-odd edge-coloring. Motivated by $[3,4,6]$, the following notion of weak-odd edge-coloring of graphs and the related weakodd chromatic index were considered in [5]. A(not necessarily proper) edgecoloring of a graph $G$ is a weak-odd edge-coloring of $G$ if each non-isolated vertex is an odd vertex in at least one of the subgraphs induced by the different color classes. In other words, for each non-isolated vertex $v \in$ $V(G)$, at least one color $c$ appears an odd number of times on $E_{G}(v)$. An obvious necessary and sufficient condition for weak-odd edge-colorability of $G$ is the absence of vertices incident only to loops. For any such graph $G$, a weak-odd edge-coloring using at most $k$ colors is referred to as a weak-odd $k$-edge-coloring of $G$, and we then say that $G$ is weak-odd $k$-edge-colorable. The weak-odd chromatic index $\chi_{\mathrm{wo}}^{\prime}(G)$ is defined as the least integer $k$ for which $G$ is weak-odd $k$-edge-colorable. The following characterization of $G$ in terms of the value of $\chi_{\mathrm{wo}}^{\prime}(G)$ was given in [5].

Theorem 1. For any connected graph $G$ whose edge set does not consist only of loops, it holds that

$$
\chi_{\mathrm{wo}}^{\prime}(G)= \begin{cases}0 & \text { if } G \text { is trivial }, \\ 1 & \text { if } G \text { is odd } \\ 3 & \text { if } G \text { is non-trivial even of odd order } \\ 2 & \text { otherwise }\end{cases}
$$


The purpose of this article is to introduce and study an analogous notion for digraphs.

Definition 1. A (not necessarily proper) edge-coloring of a digraph $D$ is said to be a weak-odd edge-coloring if for each non-isolated vertex $v \in V(D)$ at least one color $c$ is odd at $v$, i.e. satisfies the following condition:

$(C)$ if $d_{D}^{+}(v)>0$ then $c$ appears an odd number of times on the outgoing edges at $v$; and if $d_{D}^{-}(v)>0$ then $c$ appears an odd number of times on the ingoing edges at $v$.

Thus, in the particular case when $v$ is a peripheral vertex, the condition $(C)$ amounts to the appearance of $c$ an odd number of times on $E_{D}(v)$.

The minimum number of colors sufficient for a weak-odd edge-coloring of $D$ is the weak-odd chromatic index, denoted $\chi_{\text {wo }}^{\prime}(D)$. A weak-odd edgecoloring of $D$ using at most $k$ colors is referred to as a weak-odd $k$-edgecoloring, and then $D$ is said to be weak-odd $k$-edge-colorable. Hence, $\chi_{\text {wo }}^{\prime}(D)$ is the minimum integer $k$ for which $D$ is weak-odd $k$-edge-colorable.

Remark 1. We could have defined the notion weak-odd edge-coloring of a digraph $D$ alternatively - by slightly relaxing the condition for each nonisolated vertex $v \in V(D)$ :

$\left(C^{*}\right)$ if $d_{D}^{+}(v)>0$ then at least one color $c^{\prime}$ appears an odd number of times on $E_{D}^{+}(v)$; and if $d_{D}^{-}(v)>0$ then at least one color $c^{\prime \prime}$ (not necessarily the same as $\left.c^{\prime}\right)$ appears an odd number of times on $E_{D}^{-}(v)$.

But then, this 'alternative' weak-odd edge-coloring of digraphs would be just a 'disguise' of the notion weak-odd edge-coloring of bipartite graphs with equally sized partite sets. Namely, recall that a split (or bipartization) of a digraph $D$ is a bipartite graph $G$ whose partite sets $V^{+}, V^{-}$are copies of $V(D)$. For each $v \in V(D)$, there is one vertex $v^{+} \in V^{+}$and one $v^{-} \in V^{-}$. For each directed uv-edge in $D$, there is an edge with endpoints $u^{+}$and $v^{-}$ in $G$. Hence, the degrees of the vertices $v^{+}, v^{-}$in the split of $D$ are precisely the outdegree and indegree of $v$ in $D$, respectively. Furthermore, any bipartite graph $G$ with equally sized partite sets is a split of some digraph $D$, i.e. can be 'transformed' into $D$ by reversing the described procedure. Therefore, an 'alternative' weak-odd k-edge-coloring of a digraph $D$ is exactly the same thing as a weak-odd k-edge-coloring of its split $G$. This is the reason why we proceed to study the originally defined notion of weak-odd edge-coloring of digraphs.

In the next section, we prove that every digraph $D$ is weak-odd 3-edgecolorable. Moreover, we provide an example demonstrating that the upper bound $\chi_{\text {wo }}^{\prime}(D) \leq 3$ is sharp. The following section studies a related problem 
about orienting graphs. We show that every graph $G$ admits an orientation for which the obtained digraph $D$ is weak-odd 2-edge-colorable. We also solve the decision problem whether a given graph $G$ admits an orientation for which the obtained digraph $D$ is weak-odd 1-edge-colorable.

\section{Existence And a tight upper bound for $\chi_{\text {wo }}^{\prime}(D)$}

It is easy to characterize the weak-odd 1-edge-colorable digraphs $D$. Namely, $\chi_{\text {wo }}^{\prime}(D) \leq 1$ holds if and only if for every $v \in V(D)$ both $d_{D}^{+}(v), d_{D}^{-}(v)$ are odd or zero. In particular, $\chi_{\text {wo }}^{\prime}(D)=0$ holds precisely when $D$ is empty (i.e. $E(D)=\emptyset$ ). Next, we give a sufficient condition for weak-odd 2-edgecolorability of digraphs.

Lemma 1. Let $D$ be a digraph whose underlying graph is a forest. Then, $D$ is weak-odd 2-edge-colorable.

Proof. We may assume that the underlying graph of $D$ is a nonempty tree. For an arbitrary $v \in V(D)$, consider the set $E_{D}(v)$. Even if one edge $e \in E_{D}(v)$ is already colored, say by a color $c \in\{1,2\}$, this partial coloring of $E_{D}(v)$ can be turned into a (complete) coloring of $E_{D}(v)$ using the color set $\{1,2\}$ such that the color $c$ is odd at $v$. We describe how this can be done if $e \in E_{D}^{+}(v)$, and an analogous coloring procedure works for $e \in E_{D}^{-}(v)$. Since $e$ is already colored by $c$, color the rest of $E_{D}^{+}(v)$ by the other color from $\{1,2\}$. In case $v$ is a source, we are done; otherwise, select an edge $f \in E_{D}^{-}(v)$, color it by $c$, and then color the rest of $E_{D}^{-}(v)$ by the other color.

With this coloring procedure in mind, select an arbitrary vertex $v_{o} \in$ $V(D)$ and color $E_{D}\left(v_{o}\right)$ using colors from the set $\{1,2\}$ so that 1 is odd at $v_{o}$. Since the underlying graph of $D$ is connected and acyclic, as long as $E(D)$ is not fully colored, there exists a non-pendant vertex $v \in V(D)$ with just one incident edge colored so far, say by a color $c$. Apply the coloring procedure to $E_{D}(v)$ so that $c$ ends up being odd at $v$. By repeating this, we eventually construct a weak-odd 2-edge-coloring of $D$.

Clearly, the stated sufficient condition is not necessary for weak-odd 2edge-colorability (for example, any directed cycle $C$ has $\chi_{\text {wo }}^{\prime}(C)=1$ ). On the other hand, not every digraph is weak-odd 2-edge-colorable (see Fig. 1). We proceed by proving that 3 colors suffice for a weak-odd edge-coloring of every digraph $D$. Actually, we'll show a slightly stronger result which is more amenable to induction. Recall that every vertex $v$ of a digraph $D$ has precisely one of the following two types: either $v$ is an intermediate vertex (i.e. both $d_{D}^{+}(v), d_{D}^{-}(v)>0$ ), or $v$ is a peripheral vertex (a source or sink). 


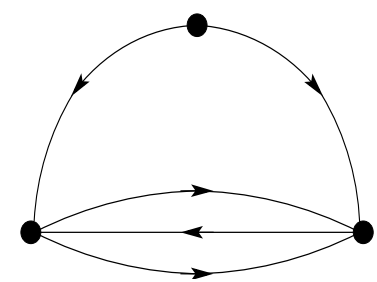

FiguRE 1. A digraph requiring 3 colors for a weak-odd edge-coloring.

Theorem 2. Every digraph admits a weak-odd edge-coloring using the color set $\{1,2,3\}$ such that at every peripheral vertex at least one of the colors 1 and 2 is odd.

Proof. For simplicity of presentation, just during this proof, we'll refer to any edge-coloring complying the statement of 2 as a good edge-coloring of the respective digraph. Suppose the theorem is false, and let $D$ be a counter-example which minimizes $|E(D)|$. Clearly, the digraph $D$ is connected, and we proceed to demonstrate several constraints which apply to it.

Claim 1. D is without directed cycles, i.e. $D$ is a directed acyclic graph.

For the sake of contradiction, suppose there exists a directed cycle $C$ in $D$. Color the edges of $C$ by 3 . Consider its edge-complement $\hat{C}=D-E(C)$. We modify the digraph $\hat{C}$ by splitting into $d_{\hat{C}}(v)$ pendant vertices every $v \in V(C)$ of degree $d_{\hat{C}}(v) \geq 2$. After this is done, let $D^{\prime}$ be the digraph obtained from $\hat{C}$. Since $D^{\prime}$ is of smaller size than $D$, there exists a good edge-coloring $\varphi^{\prime}$ of $D^{\prime}$. But, it is easily observed that we can combine $\varphi^{\prime}$ with the already given edge-coloring of $C$ to obtain a good edge-coloring $\varphi$ of $D$. The existence of $\varphi$ contradicts the choice of $D$, hence establishes the claim.

In particular, we conclude the absence of loops in the digraph $D$.

Claim 2. For every edge $e \in E(D)$ there is an endpoint having the different type in $D-e$ and $D$.

For the sake of contradiction, suppose there exists such a $v w$-edge $e$. We may take a good edge-coloring $\varphi^{\prime}$ of $D-e$. We intend to extend $\varphi^{\prime}$ to a good edge-coloring $\varphi$ of $D$. Let us observe straightaway that if at least one of the vertices $v, w$ is isolated in $D-e$, then there exists a color $c \in\{1,2\}$ such that $\varphi(e)=c$ fulfils the intention. Hence, we may assume that both 
$v$ and $w$ are non-isolated in $D-e$. Delete two colors from the set $\{1,2,3\}$ so that for each of the vertices $v$ and $w$, at least one of the deleted colors is odd at the that vertex. Say $c$ is the remaining color, and obtain $\varphi$ by setting $\varphi(e)=c$. Since each of the endpoints of $e$ has the same type in $D$ as in $D-e$, it must be that $\varphi$ is a good edge-coloring of $D$, a contradiction which proves the claim.

In particular, there are no (directed) parallel edges in $D$. Next we show that $D$ is without vertices of outdegree and indegree less than 2 .

Claim 3. There is no vertex $v \in V(D)$ such that both $d_{D}^{+}(v), d_{D}^{-}(v) \leq 1$.

Suppose the opposite, i.e. let a vertex $v$ have $d_{D}^{+}(v), d_{D}^{-}(v) \leq 1$. Assume first that $v$ is an intermediate vertex, and say $e_{1}=v_{1} v$ and $e_{2}=v v_{2}$ are the only two directed edges incident to $v$. Suppress $v$, i.e. remove it and add one directed $v_{1} v_{2}$-edge $e$. The obtained digraph $D^{\prime}$ is of smaller size than $D$, hence it admits a good edge-coloring $\varphi^{\prime}$. Construct an edge-coloring $\varphi$ of $D$ by making it agree with $\varphi^{\prime}$ on $E(D) \backslash\left\{e_{1}, e_{2}\right\}$ and setting $\varphi\left(e_{1}\right)=$ $\varphi\left(e_{2}\right)=\varphi^{\prime}(e)$. Clearly, $\varphi$ is a good edge-coloring of $D$, a contradiction.

Assume now that $v$ is a pendant vertex, and let $e$ be its incident pendant edge. Denote by $w$ the other endpoint of $e$ and consider a good edge-coloring $\varphi^{\prime}$ of the smaller digraph $D-v$. Claim 2 implies that $w$ is a peripheral vertex of $D-e$, but an intermediate vertex of $D$. Hence, at least one color $c \in\{1,2\}$ is odd at $w$ under $\varphi^{\prime}$, and we extend $\varphi^{\prime}$ to a good edge-coloring $\varphi$ of $D$ by setting $\varphi(e)=c$. This contradiction proves the claim.

Claim 4. Any $v \in V(D)$ is a peripheral vertex of $D$.

Suppose the opposite, say $v$ is an intermediate vertex of $D$, i.e. both $d_{D}^{+}(v), d_{D}^{-}(v)$ are greater than 0 . From Claim 3 , at least one of $d_{D}^{+}(v), d_{D}^{-}(v)$ is greater than 1 , say $d_{D}^{+}(v) \geq 2$. Select an $e \in E_{D}^{+}(v)$ and let $v_{1}$ be the head of $e$. Since $v$ is an intermediate vertex of $D-e$, Claim 2 implies that $d_{D}^{-}\left(v_{1}\right)=1$. Hence, Claim 3 yields $d_{D}^{+}\left(v_{1}\right) \geq 2$ and we may repeat the argument. Thus, by Claim 1, for any non-negative integer $n$ there exists a directed path $P_{n}: v v_{1} \ldots v_{n}$ in $D$. But this contradicts the finiteness of $D$, and establishes the claim.

We are now able to complete the proof. Let $T$ be a spanning tree for the underlying graph of $D$. By Lemma 1, there exists a weak-odd 2-edgecoloring $\varphi^{\prime}$ of the spanning subdigraph $D^{\prime}=D[E(T)]$ using the color set $\{1,2\}$. Since Claim 4 assures that every vertex of $D$ is peripheral, we can extend $\varphi^{\prime}$ to a good edge-coloring $\varphi$ of $D$ simply by using the color 3 for 
every edge in $E(D) \backslash E\left(D^{\prime}\right)$. But this $\varphi$ contradicts the choice of $D$, and thus proves the theorem.

Corollary 1. Every digraph $D$ is weak-odd 3-edge-colorable.

Therefore, every digraph $D$ with at least one vertex $v$ of outdegree $d_{D}^{+}(v)$ or indegree $d_{D}^{-}(v)$ even and greater than zero, satisfies $2 \leq \chi_{\text {wo }}^{\prime}(D) \leq 3$. We believe that a descriptive characterization of digraphs $D$ in terms of the value of $\chi_{\mathrm{wo}}^{\prime}(D)$ (similar to the one given for graphs in Theorem 1) is impossible. Moreover, we believe that deciding the exact value of $\chi_{\mathrm{wo}}^{\prime}(D)$ is NP-hard. Henceforth, we turn to another type of problem.

\section{A Related PROBlem about orienting GRAPhS}

In this section we consider the following question:

Question 1. Given a graph $G$ and a non-negative integer $k$, does there exist an orientation of $G$ so that the obtained digraph is weak-odd $k$-edgecolorable?

In the light of Corollary 1 , the only two nontrivial cases arise when: $k=1$ or $k=2$.

3.1. Weak-odd 2-edge-colorability. In this subsection, we provide an affirmative answer to Question 1 in the case $k=2$. For that purpose, we will need the following sufficient condition for orientability in case $k=1$.

Lemma 2. Every forest $F$ can be oriented so that the obtained digraph $D$ is weak-odd 1-edge-colorable.

Proof. We intend to orient the edges from $E(F)$ so that for every vertex $v$ of the obtained digraph $D$, both $d_{D}^{+}(v)$ and $d_{D}^{-}(v)$ are odd or zero. We may assume $F$ is a non-trivial rooted tree, say $r$ is the root. Orient first the edges from $E_{F}(r)$ as follows: if $d_{F}(r)$ is odd, then make $r$ a source; on the other hand, if $d_{F}(r)$ is even, then make the indegree of $r$ equal to 1 . Look for a non-pendant vertex $v \neq r$ for which just one edge $e \in E_{F}(v)$ is oriented so far. Since $F$ is connected and acyclic, as long as $E(F)$ is not fully oriented, there exists such a vertex $v$. The partial orientation of $E_{F}(v)$ can always be completed so that both the outdegree and indegree of $v$ are odd or zero. Namely, if $d_{F}(v)$ is odd then orient the rest of $E_{F}(v)$ consistently with the orientation of $e$ (thus $v$ becomes a peripheral vertex); on the other hand, if $d_{F}(v)$ is even, then orient the rest of $E_{F}(v)$ uniformly but inconsistently with $e$. Hence, repetition of the described procedure gives a required orientation of $F$.

The stated condition is far from being necessary, but we can use it to settle the case $k=2$ of Question 1 . 
Theorem 3. Every graph $G$ can be oriented to obtain a digraph $D$ admitting a weak-odd edge-coloring using the color set $\{1,2\}$ with every pendant edge colored by 1 .

Proof. Suppose the opposite, and let $G$ be a counter-example which minimizes $|E(G)|$. By Lemma 2, there must be a cycle $C$ in $G$. Orient the edges of $C$ consistently (i.e. turn $C$ into a directed cycle). Consider its edge-complement $\hat{C}=G-E(C)$. Modify the graph $\hat{C}$ by splitting into $d_{\hat{C}}(v)$ pendant vertices every $v \in V(C)$ of degree $d_{\hat{C}}(v) \geq 2$. After this is done, let $G^{\prime}$ be the graph obtained from $\hat{C}$. Since $G^{\prime}$ is of smaller size than $G$, it can be oriented so that the obtained digraph $D^{\prime}$ admits a weak-odd edge-coloring $\varphi^{\prime}$ using the color set $\{1,2\}$ with every pendant edge colored by 1 . Combined with the already given orientation of $C$, this gives us an orientation of $G$, and simultaneously defines an edge-coloring $\varphi$ of the obtained digraph $D$ as described in the statement (the color 2 is odd at every $v \in V(C))$. This contradicts the choice of $G$ and completes the proof.

3.2. Weak-odd 1-edge-colorability. In this subsection, we study the case $k=1$ of Question 1 . For simplicity of presentation, we often refer to any orientation of $G$ for which the respective digraph $D$ is weak-odd 1-edge-colorable as a good orientation of $G$. Moreover, the notation for the outdegree (resp. indegree) of a vertex $v \in V(D)$ is simplified to $d^{+}(v)$ (resp. $d^{-}(v)$ ). The condition for the respective digraph is that every vertex $v$ has both $d^{+}(v), d^{-}(v)$ odd or zero. Note that, unlike the case $k=2$ of Problem 1, there exist graphs which do not admit good orientations (see Fig. 2).

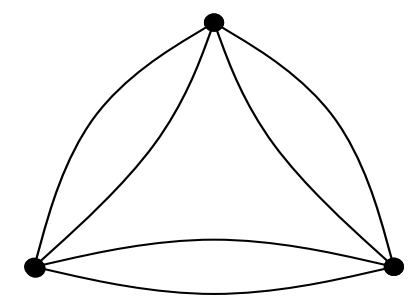

FIGURE 2. Example of a graph not admitting a good orientation.

For an arbitrary graph $G$, and let us denote by $\mathcal{O}_{G}\left(\right.$ resp. $\left.\mathcal{E}_{G}\right)$ the set of its odd (resp. even) vertices. For the particular case when $\mathcal{O}_{G}=\emptyset$, i.e. of an even graph $G$, a complete characterization in terms of the existence of a good orientation is possible. 
Theorem 4. For any even connected non-empty graph $G$, the following four statements are equivalent:

(i) For some orientation of $G$, the obtained digraph is weak-odd1-edgecolorable.

(ii) For some orientation of $G$, the set $S=\left\{v \in V(G): d^{+}(v), d^{-}(v)\right.$ are even $\}$ is even-sized.

(iii) For any orientation of $G$, the set $S=\left\{v \in V(G): d^{+}(v), d^{-}(v)\right.$ are even\} is even-sized.

(iv) The set $T=\{v \in V(G): d(v)$ is divisible by 4$\}$ is even-sized.

Proof. The implication $(i) \Rightarrow(i i)$ is obvious, since a particular orientation of $G$ is good if and only if in the corresponding digraph $D$ the set $S$ is empty. For the opposite implication, consider an orientation of $G$ for which the set $S$ (in the obtained digraph $D$ ) is even-sized. We may assume that $S$ is non-empty, say $S=\left\{u_{1}, u_{2}, \ldots, u_{2 k}\right\}$. For $i=1, \ldots, k$, select an arbitrary undirected $u_{2 i-1} u_{2 i}$-path $P_{i}$ in $D$, and reverse the orientation of every edge of $P_{i}$. By doing this, we obtain a digraph for which the corresponding $S$ is empty. This proves the implication $(i i) \Rightarrow(i)$.

Next, we demonstrate that given an arbitrary orientation of $G$, the parity of $|S|$ is an invariant of $G$, i.e. it is independent from the given orientation. Namely, select an arbitrary edge $e \in E(G)$ and reverse its orientation. Then, the size of the set $S$ either increases by 2 , stays the same, or decreases by 2 . Hence, the parity of $|S|$ remains unaltered, which proves the equivalence $(i i) \Leftrightarrow(i i i)$.

Consider now an 'Eulerian orientation' of $G$, i.e. take an Eulerian tour of $G$ and orient every traversed edge accordingly. We thus obtain a regular digraph $D$, i.e. for every vertex $v \in V(D)$ holds $d^{+}(v)=d^{-}(v)$. Hence, the set $T$ (defined in $(i v)$ ) satisfies $T=\left\{v \in V(G): d^{+}(v), d^{-}(v)\right.$ are even $\}$. Since $(i i) \Leftrightarrow(i i i)$, this yields $(i i) \Leftrightarrow(i i i) \Leftrightarrow(i v)$.

We turn next to the more general case when $\mathcal{O}_{G} \neq \emptyset$. We show that it is always possible to decide in polynomial time whether the graph $G$ admits a good orientation. First we give a necessary condition for the existence of a good orientation. Namely, it is straightforward that whenever $G$ admits one, we can orient the edges from the set

$$
E\left(\mathcal{O}_{G}\right)=\bigcup_{u \in \mathcal{O}_{G}} E(u),
$$

so that every odd vertex $u$ becomes peripheral (a source or sink). We refer to any such orientation of $E\left(\mathcal{O}_{G}\right)$ as a proper partial orientation of $G$. It is a simple matter to characterize the existence of a proper partial orientation. 
Proposition 1. The following two statements are equivalent:

(i) $G$ admits a proper partial orientation.

(ii) The induced subgraph $G\left[\mathcal{O}_{G}\right]$ is bipartite.

Proof. Suppose that $(i)$ holds. Then, there cannot be any odd cycle $C$ (a cycle of odd length) in $G$ such that $V(C) \subseteq \mathcal{O}_{G}$. Namely, if such a $C$ exists, then on an arbitrary traversing of $C$, the passed vertices are alternately sources and sinks. But this contradicts its odd length.

On the other hand, if $(i i)$ holds then we may consider a pair $X, Y$ of partite sets for $G\left[\mathcal{O}_{G}\right]$. Clearly, there exists a proper partial orientation of $G$ for which every $u \in X$ (resp. $u \in Y$ ) becomes a source (resp. sink).

Hence, a necessary condition for the existence of a good orientation of $G$ is that the subgraph $G\left[\mathcal{O}_{G}\right]$ is bipartite. In the particular case of an odd graph $G$, this necessary condition also suffices, i.e. we deduce the following.

Corollary 2. For any odd graph $G$, the next two statements are equivalent:

(i) For some orientation of $G$, the obtained digraph is weak-odd1-edgecolorable.

(ii) $G$ is bipartite.

In the remainder of this section, we restrict to connected graphs $G$ that are neither even nor odd (i.e. $\emptyset \subset \mathcal{O}_{G}, \mathcal{E}_{G} \subset V(G)$ ), and for which $G\left[\mathcal{O}_{G}\right]$ is bipartite. For such a $G$, we shall describe a simple procedure for deciding whether it admits a good orientation.

We 'decompose' $G$ into patches of two particular kinds and introduce special names for them. This is achieved by 'breaking in halves' each $e \in\left[\mathcal{O}_{G}, \mathcal{E}_{G}\right]$, i.e. every edge $e$ having endpoints of different parities (one even and one odd). By doing this, we introduce a half-edge at each of its endpoints. Observe that, after all these 'breakages in halves' are completed, we are left with partitions of the sets $\mathcal{O}_{G}$ and $\mathcal{E}_{G}$ into 'connected' subsets $\mathcal{O}_{1}, \ldots, \mathcal{O}_{t}$ and $\mathcal{E}_{1}, \ldots, \mathcal{E}_{s}$, respectively. For $j=1, \ldots, t$, the subgraph $G\left[\mathcal{O}_{j}\right]$ along with the set of half-edges incident to $\mathcal{O}_{j}$ is called the $j$-th odd patch of $G$, denoted by $X_{j}$. Similarly, for $i=1, \ldots, s$, the subgraph $G\left[\mathcal{E}_{i}\right]$ along with the set of half-edges incident to $\mathcal{E}_{i}$ is the $i$-th even patch of $G$, denoted by $Y_{i}$. Let $a_{i j}$ be the number of half-edges in $Y_{i}$ whose other halves belong to $X_{j}$.

Now, take an arbitrary proper partial orientation of $G$. This induces a (complete) orientation of every odd patch, whereas only the half-edges of every even patch are oriented. Complete the orientation of $G$ by choosing an arbitrary direction for every edge belonging to an even patch, and call it a trial orientation of $G$. In the obtained digraph, consider the sets $S_{i}=\left\{v \in \mathcal{E}_{i}: d^{+}(v), d^{-}(v)\right.$ are even $\}$, for $i=1, \ldots, s$, and let $b_{i}=\left|S_{i}\right|$. 
Observe that, by reversing the direction of any edge in $Y_{i}$ the parity of $b_{i}$ stays unaltered. On the other hand, by reversing the direction of any half-edge in $Y_{i}$ the parity of $b_{i}$ changes.

Theorem 5. The next two statements are equivalent:

(i) The trial orientation of $G$ can be modified so that the obtained digraph is weak-odd 1-edge-colorable.

(ii) The following system of linear equations is solvable over the prime field $\mathbb{Z}_{2}$.

$$
\left\{\begin{array}{c}
a_{11} x_{1}+a_{12} x_{2}+\ldots+a_{1 t} x_{t}=b_{1} \\
a_{21} x_{1}+a_{22} x_{2}+\ldots+a_{2 t} x_{t}=b_{2} \\
\ldots \ldots \\
a_{s 1} x_{1}+a_{s 2} x_{2}+\ldots+a_{s t} x_{t}=b_{s}
\end{array}\right.
$$

Proof. Suppose ( $i$ ) holds, i.e. at least one good orientation of $G$ exists. Compare one such with the trial orientation. On any odd patch $X_{j}$, either the two orientations completely match or are completely opposite. Define $\mathbf{x}_{j}$ to be equal to 0 (resp. 1) whenever the two orientation are the same (resp. opposite) on the edges of $X_{j}$. Then clearly $\mathbf{x}_{1}, \mathbf{x}_{2}, \ldots, \mathbf{x}_{t}$ is a solution of the above system of linear equations over the prime field $\mathbb{Z}_{2}$.

For the other direction of the stated equivalence, suppose ( $i i$ ) holds, and let $\mathbf{x}_{1}, \mathbf{x}_{2}, \ldots, \mathbf{x}_{t}$ be a solution of the above system of linear equations over the prime field $\mathbb{Z}_{2}$. Modify the trial orientation in accordance with the following: for every $j$ with $\mathbf{x}_{j}=1$, reverse the directions of all the edges of $G$ incident to $\mathcal{O}_{j}$. We thus obtain an orientation of $G$ such that the current set $S_{i}=\left\{v \in \mathcal{E}_{i}: d^{+}(v), d^{-}(v)\right.$ are even $\}$ is even-sized, for $i=1, \ldots, s$. On every even patch $Y_{i}$ we employ the path-reversal trick from the proof of Theorem 4. We may assume that $S_{i}$ is non-empty, say $S_{i}=\left\{u_{1}^{(i)}, u_{2}^{(i)}, \ldots, u_{2 k}^{(i)}\right\}$. For $r=1, \ldots, k$, select an arbitrary undirected $u_{2 r-1}^{(i)} u_{2 r}^{(i)}$-path $P_{r}$ in $G\left[\mathcal{E}_{i}\right]$, and reverse the orientation of every edge of $P_{r}$. After having done this 'inside' every $Y_{i}$, we end up with a good orientation of $G$.

Consequently, we are able to decide (in polynomial time) whether $G$ admits a good orientation, and if this is the case we have a way of modifying the trial orientation into a good one.

Example. It was already shown in Lemma 2 that every forest admits a good orientation. Let us give an alternative argument for this fact in the spirit of the described procedure. It is enough to consider a non-trivial tree $T$ which is not an odd graph. Since $T$ is acyclic, the induced subgraph $T\left[\mathcal{O}_{T}\right]$ is clearly bipartite. Take an arbitrary proper partial orientation of 
$T$ and extend it to a trial orientation. Denote by $A$ and $B$ the respective matrix and augmented matrix of the corresponding system of linear equations over the prime field $\mathbb{Z}_{2}$. By Kronecker-Capelli theorem (see [2]), a system of linear equations

$$
\left\{\begin{array}{c}
a_{11} x_{1}+a_{12} x_{2}+\ldots+a_{1 t} x_{t}=b_{1} \\
a_{21} x_{1}+a_{22} x_{2}+\ldots+a_{2 t} x_{t}=b_{2} \\
\ldots \ldots \\
a_{s 1} x_{1}+a_{s 2} x_{2}+\ldots+a_{s t} x_{t}=b_{s}
\end{array}\right.
$$

is compatible (i.e. solvable) over a given field if and only if the rank of the coefficient matrix $A=\left\|a_{i j}\right\|$ is equal to the rank of the augmented matrix $B$ obtained from $A$ by adding the column of free coefficients $b_{i}$. Therefore, we are left to explain why does $\operatorname{rank}(A)$ equal $\operatorname{rank}(B)$ under the given circumstances.

Since $T$ is acyclic, every $a_{i j} \in\{0,1\}$. We claim that: (1) $t>s$; and $(2)$ the rows of $A$ are linearly independent over $\mathbb{Z}_{2}$. Note that (1) and (2) together imply $\operatorname{rank}(A)=s=\operatorname{rank}(B)$. In order to prove these two claims, for $j=1, \ldots, t$ and $i=1, \ldots, s$, contract $\mathcal{O}_{j}$ into a vertex $\mathcal{O}_{j}^{\prime}$ and $\mathcal{E}_{i}$ into a vertex $\mathcal{E}_{i}^{\prime}$. The obtained graph $T^{\prime}$ is again a tree of order $s+t$. Moreover, both $\left\{\mathcal{O}_{1}^{\prime}, \ldots, \mathcal{O}_{t}^{\prime}\right\}$ and $\left\{\mathcal{E}_{1}^{\prime}, \ldots, \mathcal{E}_{s}^{\prime}\right\}$ are independent sets of vertices in $T^{\prime}$. Observe that every leaf of $T^{\prime}$ is an $\mathcal{O}_{j}^{\prime}$, and every $\mathcal{E}_{i}^{\prime}$ is an even vertex of $T^{\prime}$. The first part of this observation is obvious, and the second follows from the 'handshake lemma'. These two facts about the tree $T^{\prime}$ easily yield claim (1): it is enough to consider $T^{\prime}$ as a rooted tree with $\mathcal{E}_{1}^{\prime}$ as a root, and then count the vertices on odd (resp.) even distance from the root. To demonstrate claim (2), let $\mathcal{O}_{j}^{\prime}$ be a leaf of $T^{\prime}$ and $\mathcal{E}_{i}^{\prime}$ be its only neighbor. Then, only the $i$-th entry is non-zero in the $j$-th column of $A$, which clearly implies (2).

Acknowledgements. This work is partially supported by ARRS Program P1-0383 and by Creative Core FISNM-3330-13-500033.

\section{REFERENCES}

[1] J. A. Bondy, U. S. R. Murty, Graph Theory, Graduate Texts in mathematics, Springer, New York 244 (2008).

[2] A. Capelli, Sopra la compatibilitá o incompatibilitá di più equazioni di primo grado fra pici incognite, Revista di Mathematica 2 (1892) 54-58.

[3] J. Czap, S. Jendrol', Coloring vertices of plane graphs under restrictions given by faces, Discuss. Math. Graph Theory 293 (2009) 521-543.

[4] J. Czap, S. Jendrol', F. Kardoš, R. Soták, Facial parity edge colouring of plane pseudographs, Discrete Math. 312 (2012) 2735-2740. 
[5] M. Petruševski, A note on weak odd edge-coloring of graphs, Adv. Math. Sci. J. 4 (2015) 7-10.

[6] L. Pyber, Covering the edges of a graph by..., Graphs and Numbers, Colloquia Mathematica Societatis János Bolyai 60 (1991) 583-610.

[7] D. B. West, Introduction to Graph Theory, Pearson Education Inc. (2001). 


\title{
СЛАБО-НЕПАРНО РЕБРЕНО-БОЕЊЕ НА ДИГРАФОВИ
}

\author{
Мирко Петрушевски, Ристе Шкрековски
}

Р е зи м е

Слабо-непарно ребрено-боење на диграф $D$ претставува (не задолжително правилно) ребрено-боење при кое кај секое теме $v$ барем една боја $c$ го задоволува следниов услов: ако $d^{+}(v)>0$ тогаш $c$ се појавува непарен број пати на излезните ребра кај $v$; ако $d^{-}(v)>0$ тогаш $c$ се појавува непарен број пати на влезните ребра кај $v$. Минималниот број бои доволни за слабо-непарно ребрено-боење на $D$ е негов слабо-непарен хроматски индекс, со ознака $\chi_{\text {wo }}^{\prime}(D)$. Во оваа статија докажуваме дека за секој диграф $D$ важи $\chi_{\text {wo }}^{\prime}(D) \leq 3$, при што равенство е достижно. Проучуваме кога даден граф дозволува ориентација при која добиениот диграф е слабо-непарно 1-ребрено-обојлив. Покажуваме и дека секој граф дозволува ориентација при која добиениот диграф е слабо-непарно 2-ребрено-обојлив.

Department of Mathematics and Informatics, Faculty of Mechanical EngineEring - Skopje, Republic of Macedonia.

E-mail address: mirko.petrushevski@gmail.com

Institute of Mathematics, Physics and Mechanics, 1000 Ljubljana, Slovenia \& Faculty of Information Studies, 8000 Novo mesto, Slovenia \& UniverSity of Primorska, FAMNIT, 6000 Koper, Slovenia.

E-mail address: skrekovski@gmail.com 